\title{
Riqueza Vegetal da Bacia Hidrográfica do Igarapé da Fortaleza, AP-010, Amapá, Brasil
}

\author{
Vegetation Richness of the Igarapé da Fortaleza Hydrographic Basin, AP-010, Amapá, Brazil \\ Riqueza Vegetal de la Cuenca Hidrográfica de Igarapé da Fortaleza, AP-010, Amapá, Brasil
}

Recebido: 30/09/2021 | Revisado: 09/10/2021 | Aceito: 18/10/2021 | Publicado: 20/10/2021

Patrick de Castro Cantuária

ORCID: https://orcid.org/0000-0002-3676-7866 Instituto de Pesquisas Científicas e Tecnológicas do Estado do Amapá, Brasil

E-mail: patrickcantuaria@gmail.com

Tonny David Santiago Medeiros

ORCID: https://orcid.org/0000-0002-0891-1584 Instituto de Pesquisas Científicas e Tecnológicas do Estado do Amapá, Brasil

E-mail: tonnyiepa@gmail.com

Mellissa Souza Sobrinho

ORCID: https://orcid.org/0000-0001-6397-939X Universidade Federal do Amapá, Brasil

E-mail: mellissasobrinho@unifap.br

Raullyan Borja Lima e Silva

ORCID: https://orcid.org/0000-0003-4104-5611 Instituto de Pesquisas Científicas e Tecnológicas do Estado do Amapá, Brasil

E-mail: raullyanborja@gmail.com

Salustiano Vilar da Costa Neto

ORCID: https://orcid.org/0000-0002-1459-3658 Instituto de Pesquisas Científicas e Tecnológicas do Estado do Amapá, Brasil

E-mail: salucostaneto@gmail.com

Camila Barbosa de Araújo

ORCID: https://orcid.org/0000-0001-9251-943X

Instituto de Botânica de São Paulo, Brasil

E-mail: camiaraujo.ba@gmail.com

Mayara Fabiana de Melo Furtado

ORCID: https://orcid.org/0000-0003-1832-5025

Universidade Federal do Amapá, Brasil

E-mail: mayarafabiana@gmail.com

Amauri Herbert Krahl

ORCID: https://orcid.org/0000-0001-7862-9624 Instituto Nacional de Pesquisas da Amazônia, Brasil

E-mail: amauri.her.krahl@gmail.com

Alana Carine Sobrinho Soares

ORCID: https://orcid.org/0000-0003-0464-1908

Universidade do Estado do Amapá, Brasil

E-mail: alanaufra@hotmail.com

Ana Luzia Ferreira Farias

ORCID: https://orcid.org/0000-0002-5338-6598

Universidade do Estado do Amapá, Brasil

E-mail: analuziafarias@gmail.com

Plínio Marcos Bahia Potyguara

ORCID: https://orcid.org/0000-0002-0512-8340 Instituto de Pesquisas Científicas e Tecnológicas do Estado do Amapá, Brasil E-mail: pliniopotyguara@gmail.com

Sheylla Susan Moreira da Silva de Almeida ORCID: https://orcid.org/0000-0002-7687-8288

Universidade Federal do Amapá, Brasil

E-mail: sheyllasusan@yahoo.com.br

Juliana Eveline dos Santos Farias

ORCID: https://orcid.org/0000-0003-0121-2868 Instituto Federal de Educação, Ciência e Tecnologia do Amapá, Brasil E-mail: juliana.farias@ifap.edu.br

\section{Resumo}

O objetivo desta pesquisa é conhecer a diversidade de espécies vegetais coletadas na abrangência da Bacia Hidrográfica do Igarapé da Fortaleza, entre os municípios de Santana e Macapá, Estado do Amapá. A partir da organização de registros digitais de dados de espécies coletadas e tombadas em herbários nacionais e internacionais, 
foram extraídos os registros coletados no Estado do Amapá, compreendendo a abrangência da área de interesse da pesquisa. A composição florística foi avaliada pela riqueza dos indivíduos distribuídos em gêneros e espécies. As famílias foram organizadas de acordo com o APG IV e a atualização de nomenclatura e verificação de hábito de vida foram realizadas por meio de consulta aos sites Flora do Brasil 2020 e Tropicos. Foram registradas 501 amostras tombadas em 18 herbários, sendo 11 nacionais e sete internacionais, destas, 235 depositadas no Herbário Amapaense. Registraram-se 245 espécies pertencentes a 60 famílias e 169 gêneros. Fabaceae foi a família com o maior número de espécies registradas, seguida de Poaceae e Orchidaceae, e o hábito mais frequente foi herbáceo. Conclui-se que a diversidade florística da Bacia Hidrográfica do Igarapé da Fortaleza é bastante significativa, mas mesmo com expressiva diversidade registrada ainda há necessidade de estudos adicionais para ampliação do conhecimento da diversidade existente.

Palavras-chave: Áreas úmidas; Coleções botânicas; Diversidade; Flora urbana.

\begin{abstract}
The objective of this research is to know the diversity of plant species collected in the scope of the Igarapé da Fortaleza Hydrographic Basin, between the municipalities of Santana and Macapá, State of Amapá. From the organization of digital data records of species collected and registered in national and international herbaria, the records collected in the State of Amapá were extracted, comprising the scope of the research interest area. The floristic composition was evaluated by the richness of individuals distributed in genera and species. The families were organized according to the APG IV and the nomenclature update and verification of life habits were carried out by consulting the Flora do Brasil 2020 and Tropicos websites. A total of 501 samples were registered in 18 herbariums, 11 of which were national and seven internationals, of which 235 were deposited in the Herbarium Amapaense. 245 species belonging to 60 families and 169 genera were recorded. Fabaceae was the family with the largest number of species recorded, followed by Poaceae and Orchidaceae, and the most frequent habit was herbaceous. It is concluded that the floristic diversity of the Igarapé da Fortaleza Hydrographic Basin is quite significant, but even with the expressive diversity recorded, there is still a need for additional studies to expand the knowledge of the existing diversity.
\end{abstract}

Keywords: Wetlands; Botanical collections; Diversity; Urban flora.

\title{
Resumen
}

El objetivo de esta investigación es conocer la diversidad de especies vegetales recolectadas en el ámbito de la Cuenca Hidrográfica de Igarapé da Fortaleza, entre los municipios de Santana y Macapá, Estado de Amapá. De la organización de registros de datos digitales de especies recolectadas y registradas en herbarios nacionales e internacionales, se extrajeron los registros recolectados en el Estado de Amapá, que comprenden el alcance del área de interés de investigación. La composición florística fue evaluada por la riqueza de individuos distribuidos en géneros y especies. Las familias se organizaron según la APG IV y la actualización de nomenclatura y verificación de hábitos de vida se realizó mediante la consulta de los sitios web Flora do Brasil 2020 y Tropicos. Se registraron un total de 501 muestras en 18 herbarios, de los cuales 11 fueron nacionales y siete internacionales, de los cuales 235 fueron depositados en el Herbario Amapaense. Se registraron 245 especies pertenecientes a 60 familias y 169 géneros. Fabaceae fue la familia con mayor número de especies registradas, seguida de Poaceae y Orchidaceae, y el hábito más frecuente fue el herbáceo. Se concluye que la diversidad florística de la Cuenca Hidrográfica de Igarapé da Fortaleza es bastante significativa, pero incluso con la diversidad expresiva registrada, todavía se necesitan estudios adicionales para ampliar el conocimiento de la diversidad existente.

Palabras clave: Humedales; Colecciones botánicas; Diversidad; Flora urbana.

\section{Introdução}

Ao considerar a Amazônia e sua extensão florestal existem muitas lacunas quanto a realização de inventários biológicos em determinados domínios fitogeográficos. A organização de inventários botânicos na Região Amazônica começou a ser feita há mais de dois séculos e tem contribuído para identificar milhares de espécies. Apesar desse volume de informações, essa região ainda é pouco conhecida quando comparada a outras e essas lacunas só podem ser preenchidas com a sistematização de coletas botânicas e a identificação dos espécimes, com posterior incorporação a coleções de referência.

O Estado do Amapá contempla cobertura vegetal diversa, que abrange florestas de terra firme, várzea, campos inundados, savanas e áreas de ressaca, terminologia local para áreas úmidas com ou sem inundação total ou parcial (Pereira et al., 2011; Takiyama et al., 2004). Possui uma das menores taxas de desmatamento do Brasil e é o estado mais bem conservado do país, com aproximadamente $70 \%$ de sua área protegida em 12 Unidades de Conservação, divididas em Unidades de 
Proteção Integral e outras de Uso Sustentável, que integram o Corredor de Biodiversidade do Amapá, tendo como principal unidade o Parque Nacional Montanhas do Tumucumaque (CIB, 2009).

A Bacia Hidrográfica do Igarapé da Fortaleza compreende área de $\mathrm{x}$ ha, que abrange do Igarapé da Fortaleza, no município de Santana, até a Lagoa dos Índios, no município de Macapá, sendo perpassada pela Rodovia Josmar Chaves Pinto que corresponde ao perímetro urbano da AP-010 (fonte). Nessa região, caracterizada principalmente por possuir ambientes com florestas de várzea e áreas de ressaca, que mantêm relação direta com os cursos d'água, há grande ocupação e atividade humana, para moradia e subsistência (fonte).

Como exemplo, a Área de Proteção Ambiental da Fazendinha (APA da Fazendinha), concentra o desembarque de mercadorias e pessoas, mas também apresenta graus significativos de modificação de sua vegetação, ligada à exploração dos recursos, seja para a ocupação humana ou o estabelecimento de empreendimentos de grande porte (fonte). O principal curso d'água, ligado às principais áreas ressaca de Macapá e Santana, indica significativo grau de comprometimento e degradação ambiental, onde a retirada das matas ciliares, erosão das margens, assoreamento intensivo e urbanização desordenada, trazem reflexos negativos para a qualidade da água da bacia hidrográfica (Cunha et al., 2004).

Embora exista um número significativo de áreas protegidas, preservar a diversidade vegetal ainda é um desafio, uma vez que as informações quanto à composição florística de uma região são fundamentais para que se saiba como preservar, no entanto, as existentes não estão organizadas, de modo que não se sabe quantas e quais espécies ocorrem (Cantuária et al., 2017; Pereira et al., 2011). Como consequência, ações de preservação ou de desenvolvimento local têm poucos parâmetros biológicos disponíveis para a determinação de suas atividades.

As coleções científicas botânicas são importantes nesse contexto, pois correspondem a fontes de dados materiais e documentais que validam as informações sobre a diversidade e a riqueza da cobertura vegetal de uma região, nas quais se mantêm registros preservados de espécimes ou informações sobre eles, armazenados em espaços específicos, os herbários. Estes representam elementos primordiais para pesquisas taxonômicas, florísticas e ecológicas, dentre outros, pois é possível extrair informações válidas quanto às espécies que compõem a flora de um estado, região ou local, distribuição geográfica, bem como possibilitam apontar áreas pouco amostradas, evidenciando a necessidade de coletas (Peixoto et al., 2009; Siqueira et al., 2012).

Ainda que a diversidade florística do Estado do Amapá seja reconhecida como uma das mais significativas da Amazônia brasileira, pelo estado de conservação de suas florestas, as informações disponíveis no Herbário Amapaense (HAMAB), apesar de, quase em sua totalidade, digitalizada, não estão sistematizadas a ponto de permitir a determinação de sua representatividade em nível de diversidade local, de ambientes amostrados e de suficiência amostral. Considerando ainda que muitos registros foram efetuados somente em outros herbários.

O objetivo deste estudo foi conhecer a diversidade de espécies vegetais coletadas na abrangência da Bacia Hidrográfica do Igarapé da Fortaleza, entre os municípios de Santana e Macapá, no Estado do Amapá. A importância desta pesquisa relaciona-se à disponibilização de informações que contribuirão para a elaboração e execução de políticas públicas de conservação de áreas úmidas na região e como subsídio para novos estudos.

\section{Metodologia}

\subsection{Caracterização da Área de Estudo}

A pesquisa foi realizada no Estado do Amapá, território que integra o bioma da Amazônia, localizado no extremo norte do Brasil. Com limites a noroeste e norte da Guiana Francesa e a noroeste e leste do Oceano Atlântico, é cercado pelo Rio Amazonas, entre os paralelos de $0^{\circ}$ e $03^{\circ} \mathrm{S}$ e meridianos $50^{\circ}$ e $54^{\circ} \mathrm{W}$, fazendo fronteira com Pará e Suriname (Abreu et al., 2014; Brasil, 1943). O clima do estado é caracterizado como quente e úmido com duas estações bem definidas, verão 
(menos chuvoso) e inverno (mais chuvoso), o que favorece a cobertura vegetal diversa, com predomínio de Floresta Ombrófila Densa de Terra Firme e Aluvial e Savana Amazônica ou Cerrado (Instituto Brasileiro de Geografia e Estatística [IBGE], 2012; Neves, 2012).

As informações levantadas correspondem especificamente à Bacia Hidrográfica do Igarapé da Fortaleza, que compreende área entre os municípios de Macapá e Santana, AP-010 (Figura 1).

Figura 1 - Mapa de localização da AP-010, com destaque para os municípios de Santana e Macapá, onde está situada a Bacia Hidrográfica do Igarapé da Fortaleza, Amapá, Brasil, 2021.

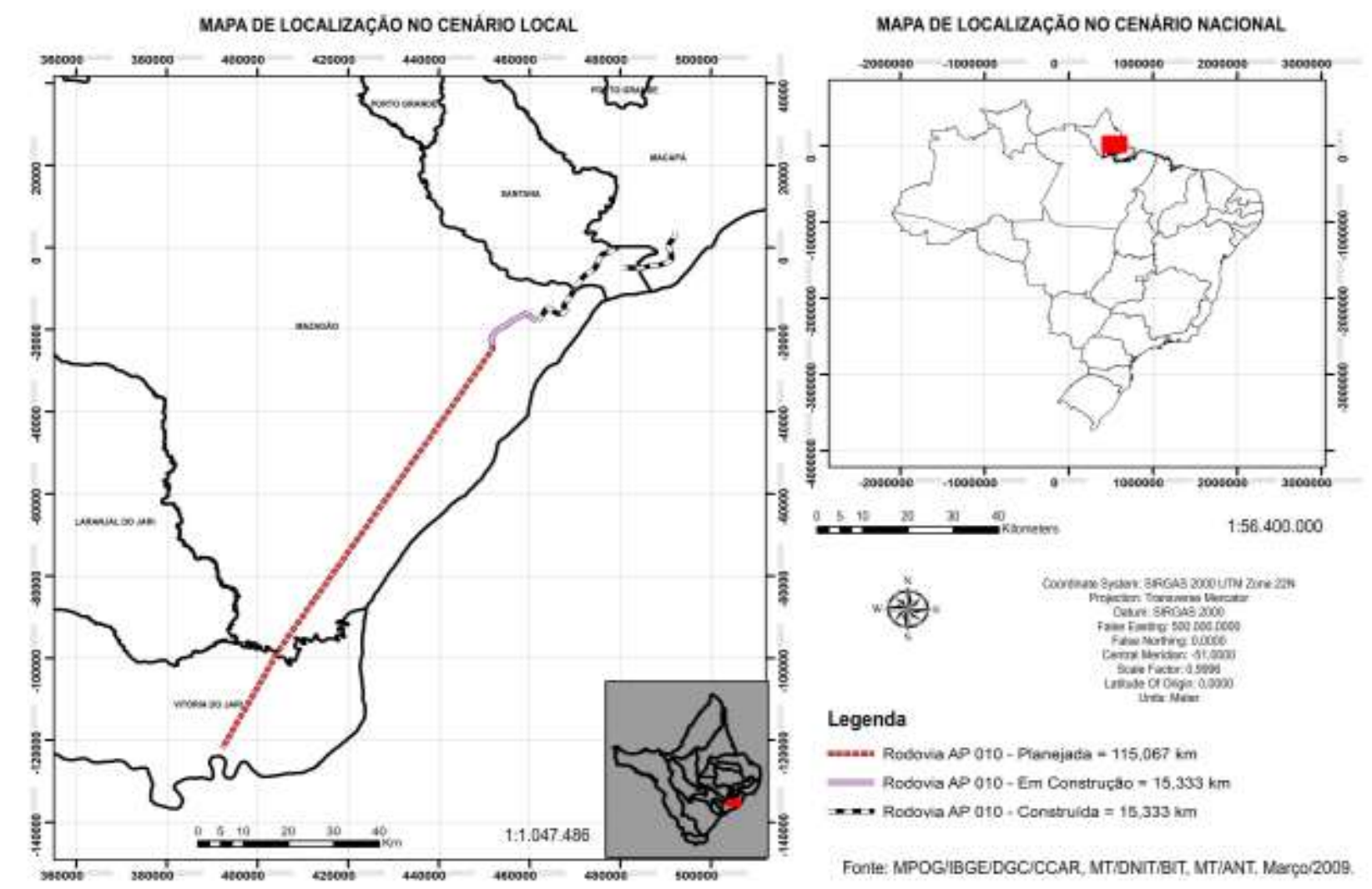

Fonte: Autores (2021).

A Bacia do Igarapé da Fortaleza é influenciada pela dinâmica das águas do Rio Amazonas e também pelo regime de precipitação. Nela encontram-se diferentes tipologias florestais, desde florestas de várzea, terra firme, cerrado, campos abertos e áreas de ressaca (Takiyama et al., 2004). As medições demonstram que a influência das marés sobre os corpos hídricos se comporta de forma diferenciada, de acordo com a sazonalidade e a localização ao longo da bacia (Takiyama et al., 2012).

\subsection{Coleta e Análise de Dados}

A partir dos dados dos registros digitais em que constam informações de amostras de espécies coletadas e tombadas no HAMAB e em outros herbários nacionais e internacionais, e atribuídos seus acrônimos (Thiers, 2021), foram extraídos apenas os registros coletados no Estado do Amapá. Os dados obtidos por meio das buscas nos herbários foram organizados em planilha eletrônica Excel (2019).

Foram verificados e corrigidos campos (p. ex. "família", "gênero" e "espécie") com erros de digitação, registros duplicados e demais erros relacionados à localidade de coleta e grafia das nomenclaturas. Por fim, foram levantadas informações gerais em cada registro para permitir inserir campos adicionais na planilha quando necessário, como "formas de 
vida", para descrever as formas de vida (árvore, arbusto, erva, epífita...) de cada espécie. Todos os dados foram tabulados e organizados da seguinte forma: família, espécie, forma de vida, coletor e número de coleta, coleção de referência e coordenadas geográficas.

Para a construção da lista das espécies foram consultados os sítios speciesLink (SpeciesLink network, 2021) e Flora do Brasil 2020 (Flora do Brasil 2020, 2021). As famílias foram organizadas de acordo com o Angiosperm Phylogene Group (APG IV, 2016) e a atualização de nomenclatura e verificação da forma de vida, foram realizadas por meio de consulta aos sítios Tropicos (Tropicos.org, 2021) e Flora do Brasil 2020 (Flora do Brasil 2020, 2021).

\section{Resultados e Discussão}

Foram registradas 501 amostras tombadas em 18 herbários, sendo 11 nacionais (BHCB, CEN, HAMAB, HUEFS, IAN, INPA, RB, SPF, UB, UFRN e UFRR) e sete internacionais (ASU, CAS, K, MO, NY, NBR e US), destas, 235 depositadas no HAMAB.

Registraram-se 245 espécies pertencentes a 60 famílias e 169 gêneros, tendo sido 38 espécies determinas apenas em nível de gênero e cinco indeterminadas. Fabaceae foi a família com o maior número de espécies registradas (49), seguida de Poaceae (27) e Orchidaceae (21) (Tabela 1).

Tabela 1 - Listagem das espécies registradas na Bacia Hidrográfica do Igarapé da Fortaleza, Macapá/Santana, Amapá, 2021.

\begin{tabular}{|c|c|c|c|c|}
\hline Família & Espécie & Forma de Vida & Coletor e no & Herbário \\
\hline Achariaceae & Lindackeria pauciflora Benth. & Arbusto, Árvore & B.V. Rabelo 2841 & HAMAB \\
\hline Anacardiaceae & Tapirira guianensis Aubl. & Árvore & $\begin{array}{l}\text { S.A. Mori } 15705 ; \mathrm{R} . \\
\text { Nonato } 5\end{array}$ & HAMAB, NY, US \\
\hline Annonaceae & Annona montana Macfad. & Árvore & B.V. Rabelo 1436 & HAMAB \\
\hline Apocynaceae & $\begin{array}{l}\text { Mandevilla scabra (Hoffmanns. ex } \\
\text { Roem. \& Schult.) K.Schum. }\end{array}$ & Liana/volúvel/trepadeira & B.V. Rabelo 2259 & HAMAB, NY, US \\
\hline \multirow{4}{*}{ Araceae } & Monstera adansonii Schott & $\begin{array}{c}\text { Erva, } \\
\text { Liana/volúvel/trepadeira }\end{array}$ & L.S.B. Calazans 515 & $\mathrm{RB}$ \\
\hline & Philodendron muricatum Schott & $\begin{array}{c}\text { Erva, } \\
\text { Liana/volúvel/trepadeira } \\
\end{array}$ & L.A. Pereira 80 & HАMAB \\
\hline & Urospatha sagittifolia (Rudge) Schott & Erva & L.A. Pereira 165 & HAMAB \\
\hline & Xanthosoma acutum E.G.Gonç. & Erva & E.G. Gonçalves 555 & UB \\
\hline Araliaceae & $\begin{array}{l}\text { Schefflera morototoni (Aubl.) Maguire, } \\
\text { Steyerm. \& Frodin }\end{array}$ & Árvore & B.V. Rabelo 2296 & HAMAB, NY \\
\hline \multirow{4}{*}{ Arecaceae } & Euterpe oleracea Mart. & Erva & $\begin{array}{l}\text { M. Groppo-Junior } \\
1137\end{array}$ & HAMAB \\
\hline & Mauritia flexuosa L.f. & Erva & $\begin{array}{l}\text { M. Groppo-Junior } \\
1127\end{array}$ & HАMAB \\
\hline & Mauritiella armata (Mart.) Burret & Erva & $\begin{array}{c}\text { M. Groppo-Junior } \\
1128 \\
\end{array}$ & HAMAB \\
\hline & Oenocarpus bacaba Mart. & Erva & $\begin{array}{l}\text { B.V. Rabelo 2301, } \\
\text { 2302; M. Groppo- } \\
\text { Junior } 1136\end{array}$ & HАMAB \\
\hline Bignoniaceae & $\begin{array}{l}\text { Adenocalymma neoflavidum } \\
\text { L.G.Lohmann }\end{array}$ & $\begin{array}{c}\text { Arbusto, } \\
\text { Liana/volúvel/trepadeira } \\
\end{array}$ & S.A. Mori 15755 & NY \\
\hline \multirow[b]{2}{*}{ Bixaceae } & Cochlospermum regium (Schrank) Pilg. & Arbusto, Subarbusto & B.V. Rabelo 500 & NY, RBR \\
\hline & $\begin{array}{l}\text { Cochlospermum vitifolium (Willd.) } \\
\text { Spreng. }\end{array}$ & Arbusto, Árvore & S.A. Mori 15751 & NY \\
\hline \multirow{2}{*}{ Boraginaceae } & Cordia bicolor A.DC & Árvore & B.V. Rabelo 2295 & NY, HUEFS \\
\hline & Cordia multispicata Cham. & Arbusto, Árvore & L.A. Pereira 62 & HAMAB \\
\hline
\end{tabular}




\begin{tabular}{|c|c|c|c|c|}
\hline \multirow{3}{*}{ Bromeliaceae } & $\begin{array}{l}\text { Aechmea mertensii (G.Mey.) Schult. \& } \\
\text { Schult.f. }\end{array}$ & Erva & $\begin{array}{l}\text { L.A. Pereira 84; } \\
\text { B.V. Rabelo } 1443 \\
\end{array}$ & HAMAB \\
\hline & Araeococcus micranthus Brongn. & Erva & R.A. Pontes 1190 & UFRN \\
\hline & Bromelia balansae $\mathrm{Mez}$ & Erva & S.A. Mori 15748 & NY \\
\hline \multirow{3}{*}{ Burseraceae } & $\begin{array}{l}\text { Crepidospermum goudotianum (Tul.) } \\
\text { Triana \& Planch. }\end{array}$ & Arbusto, Árvore & $\begin{array}{c}\text { B.V. Rabelo 2002, } \\
\text { 2497; D.C. Daly } \\
\text { 3775; S.A. Mori } \\
\text { 17406 }\end{array}$ & $\begin{array}{l}\text { HAMAB, NY, US, } \\
\text { HUEFS }\end{array}$ \\
\hline & $\begin{array}{l}\text { Protium heptaphyllum (Aubl.) } \\
\text { Marchand }\end{array}$ & Arbusto, Árvore & $\begin{array}{l}\text { D.C. Daly } 3774, \\
3776\end{array}$ & $\begin{array}{l}\text { HAMAB, NY, US, } \\
\text { HUEFS }\end{array}$ \\
\hline & Trattinnickia rhoifolia Willd. & Árvore & B.V. Rabelo 2298 & NY, US \\
\hline Calymperaceae & Calymperes palisotii Schwägr. & Erva & S.A. Mori 17413 & HAMAB \\
\hline Cannabaceae & Trema micrantha (L.) Blume & Arbusto, Árvore & $\begin{array}{l}\text { R.L. Froés } 27491 ; \\
\quad \text { R. Nonato } 3\end{array}$ & IAN, HAMAB \\
\hline \multirow{8}{*}{ Chrysobalanaceae } & Hirtella bicornis Mart. \& Zucc. & Arbusto, Árvore & B.V. Rabelo 2486 & $\mathrm{~K}, \mathrm{US}$ \\
\hline & Hirtella eriandra Benth. & Arbusto, Árvore & B.V. Rabelo 2477 & $\mathrm{~K}, \mathrm{US}$ \\
\hline & Hirtella paraensis Prance & Arbusto, Árvore & B.V. Rabelo 2285 & $\mathrm{~K}$ \\
\hline & Licania kunthiana Hook.f. & Árvore & $\begin{array}{l}\text { B.V. Rabelo 2491, } \\
1686\end{array}$ & NY, K, US \\
\hline & Licania minutiflora (Sagot) Fritsch & Árvore & B.V. Rabelo 2475 & NY, K, US \\
\hline & $\begin{array}{l}\text { Licania octandra (Hoffmanns. ex Roem. } \\
\text { \& Schult.) Kuntze }\end{array}$ & Árvore & $\begin{array}{l}\text { B.V. Rabelo 2275, } \\
2478,2482,2480\end{array}$ & US, K, NY \\
\hline & \begin{tabular}{|l|} 
Licania sprucei (Hook.f.) Fritsch. \\
\end{tabular} & Árvore & B.V. Rabelo 230 & NY \\
\hline & Parinari excelsa Sabine & Árvore & B.V. Rabelo 2773 & NY \\
\hline \multirow{4}{*}{ Combretaceae } & Buchenavia parvifolia Ducke & Árvore & B.V. Rabelo 2277 & NY \\
\hline & $\begin{array}{l}\text { Buchenavia tetraphylla (Aubl.) } \\
\text { R.A.Howard }\end{array}$ & Árvore & B.V. Rabelo 2843 & HAMAB \\
\hline & Combretum laxum Jacq. & $\begin{array}{c}\text { Arbusto, Árvore, } \\
\text { Liana/volúvel/trepadeira }\end{array}$ & B.V. Rabelo 1428 & HAMAB \\
\hline & Terminalia dichotoma G.Mey. & Árvore & B.V. Rabelo 1438 & HAMAB \\
\hline Connaraceae & Connarus perrottetii (DC.) Planch. & Árvore & B.V. Rabelo 2289 & HAMAB, NY \\
\hline Convolvulaceae & Maripa reticulata Ducke & Liana/volúvel/trepadeira & S.A. Mori 15754 & NY \\
\hline Crassulaceae & Bryophyllum pinnatum (Lam.) Oken & Erva & B.V. Rabelo 1437 & HAMAB \\
\hline \multirow{6}{*}{ Cyperaceae } & Bulbostylis conifera (Kunth) Beetle & Erva & S.A. Mori 15749 & NY \\
\hline & $\begin{array}{l}\text { Bulbostylis junciformis (Kunth) } \\
\text { C.B.Clarke }\end{array}$ & Erva & S.A. Mori 15719 & NY, K \\
\hline & Bulbostylis truncata (Nees) M.T.Strong & Erva & S.A. Mori 15716 & NY, K \\
\hline & Rhynchospora cephalotes (L.) Vahl & Erva & $\begin{array}{l}\text { S.A. Mori 17418, } \\
15753\end{array}$ & HAMAB, NY, K \\
\hline & $\begin{array}{l}\text { Rhynchospora holoschoenoides (Rich.) } \\
\text { Herter }\end{array}$ & Erva & S.A. Mori 17400 & HAMAB, NY \\
\hline & $\begin{array}{l}\text { Rhynchospora trispicata (Nees) Schrad. } \\
\text { ex Steud. }\end{array}$ & Erva & S.A. Mori 17394 & NY \\
\hline \multirow{3}{*}{ Erythroxylaceae } & Erythroxylum ligustrinum DC. & Arbusto, Árvore & B.V. Rabelo 2513 & NY \\
\hline & Erythroxylum squamatum $\mathrm{Sw}$. & Arbusto, Árvore & R.L. Froés 27497 & UB, IAN \\
\hline & Erythroxylum suberosum A.St.-Hil. & $\begin{array}{c}\text { Arbusto, Árvore, } \\
\text { Subarbusto }\end{array}$ & S.A. Mori 15706 & $\begin{array}{l}\text { HAMAB, NY, K, } \\
\text { US }\end{array}$ \\
\hline \multirow{4}{*}{ Euphorbiaceae } & $\begin{array}{l}\text { Alchorneopsis floribunda (Benth.) } \\
\text { Müll.Arg. }\end{array}$ & Árvore & B.V. Rabelo 2829 & HAMAB \\
\hline & Dalechampia affinis Müll.Arg. & Liana/volúvel/trepadeira & S.A. Mori 15723 & HAMAB, NY \\
\hline & Dalechampia parvibracteata Lanj. & Liana/volúvel/trepadeira & B.V. Rabelo 1424 & HAMAB \\
\hline & $\begin{array}{l}\text { Hevea brasiliensis (Willd. ex A.Juss.) } \\
\text { Müll.Arg. }\end{array}$ & Árvore & B.V. Rabelo 1447 & HАMAB \\
\hline
\end{tabular}




\begin{tabular}{|c|c|c|c|}
\hline Margaritaria nobilis L.f. & Arbusto & $\begin{array}{l}\text { B.V. Rabelo 1426, } \\
493\end{array}$ & HAMAB \\
\hline Richeria grandis Vahl & Arbusto, Árvore & $\begin{array}{l}\text { B.V. Rabelo 1892, } \\
2817 \\
\end{array}$ & HAMAB \\
\hline Aeschynomene brasiliana (Poir.) DC. & $\begin{array}{l}\text { Arbusto, Erva, } \\
\text { Subarbusto }\end{array}$ & S.A. Mori 15714 & HAMAB \\
\hline Albizia pedicellaris (Dc.) L.Rico & Árvore & B.V. Rabelo 2284 & HAMAB \\
\hline Anadenanthera peregrina (L.) Speg. & Árvore & S.A. Mori 15704 & HAMAB \\
\hline Andira inermis (Wright) DC. & Árvore & B.V. Rabelo 1433 & HAMAB, NY \\
\hline Bauhinia guianensis Aubl. & Liana/volúvel/trepadeira & B.V. Rabelo 2303 & HAMAB \\
\hline Centrosema brasilianum (L.) Benth. & Liana/volúvel/trepadeira & N.M.S. Costa 2044 & CEN \\
\hline Centrosema rotundifolium Mart. & $\begin{array}{c}\text { Erva, } \\
\text { Liana/volúvel/trepadeira, } \\
\text { Subarbusto }\end{array}$ & P. Ledoux 56245 & IAN, RB \\
\hline Chamaecrista nictitans (L.) Moench & Erva, Subarbusto & N.M.S. Costa 2045 & CEN \\
\hline $\begin{array}{l}\text { Chamaecrista patellaria (DC. ex } \\
\text { Collad.) Greene }\end{array}$ & Erva, Subarbusto & N.M.S. Costa 2045 & CEN \\
\hline Clitoria arborea Benth. & Árvore & $\begin{array}{l}\text { R.L. Froés } 27487 ; \\
\text { S.A. Mori } 15718 \\
\end{array}$ & NY, IAN \\
\hline Clitoria fairchildiana R.A.Howard & Árvore & L.A. Pereira 61 & HAMAB \\
\hline Clitoria guianensis (Aubl.) Benth. & Erva, Subarbusto & R. Nonato 22 & HAMAB \\
\hline Dalbergia monetaria L.f. & $\begin{array}{c}\text { Arbusto, } \\
\text { Liana/volúvel/trepadeira }\end{array}$ & B.V. Rabelo 1440 & HAMAB \\
\hline Desmodium appressipilum B.G.Schub. & Subarbusto & B.V. Rabelo 1324 & HAMAB \\
\hline Desmodium barbatum (L.) Benth. & Subarbusto & S.A. Mori 15708 & HAMAB, NY \\
\hline Dipteryx odorata (Aubl.) Willd. & Árvore & $\begin{array}{l}\text { B.V. Rabelo 3278; } \\
\text { J. Freitas 3; B.V. } \\
\text { Rabelo } 2263\end{array}$ & HAMAB \\
\hline Eriosema crinitum (Kunth) G.Don & $\begin{array}{l}\text { Arbusto, Erva, } \\
\text { Subarbusto }\end{array}$ & R.L. Froés 27498 & INPA \\
\hline Eriosema violaceum (Aubl.) G.Don & Erva, Subarbusto & S.A. Mori 15712 & HAMAB, NY \\
\hline Erythrina fusca Lour. & Árvore & B.V. Rabelo 1408 & HAMAB \\
\hline Hymenolobium petraeum Ducke & Árvore & B.V. Rabelo 2484 & HAMAB, NY \\
\hline Indigofera suffruticosa Mill. & Arbusto, Subarbusto & $\begin{array}{l}\text { B.V. Rabelo 498; P. } \\
\quad \text { Ledoux } 1108\end{array}$ & HAMAB, IAN \\
\hline Inga alba (Sw.) Willd. & Árvore & $\begin{array}{l}\text { B.V. Rabelo 2279, } \\
2280\end{array}$ & НАMAB \\
\hline Inga microcalyx Benth. & Árvore & B.V. Rabelo 1431 & HAMAB \\
\hline Inga semialata (Vell.) C.Mart. & Árvore & S.A. Mori 15722 & HAMAB, NY \\
\hline Machaerium lunatum (L.f.) Ducke & $\begin{array}{c}\text { Arbusto, Árvore, } \\
\text { Liana/volúvel/trepadeira }\end{array}$ & B.V. Rabelo 1422 & HAMAB \\
\hline Mimosa pigra $\mathrm{L}$. & Arbusto & B.V. Rabelo 491 & HAMAB \\
\hline Ormosia paraensis Ducke & Árvore & $\begin{array}{l}\text { B.V. Rabelo 2281, } \\
2285\end{array}$ & HAMAB, K, NY \\
\hline $\begin{array}{l}\text { Senna chrysocarpa (Desv.) H.S. Irwin \& } \\
\text { Barneby }\end{array}$ & $\begin{array}{c}\text { Arbusto, } \\
\text { Liana/volúvel/trepadeira }\end{array}$ & $\begin{array}{l}\text { R.L. Froés 27489, } \\
497 \\
\end{array}$ & IAN, NY \\
\hline $\begin{array}{l}\text { Senna latifolia (G.Mey.) H.S.Irwin \& } \\
\text { Barneby }\end{array}$ & Arbusto, Subarbusto & B.V. Rabelo 2524 & NY \\
\hline $\begin{array}{l}\text { Senna silvestris (Vell.) H.S.Irwin \& } \\
\text { Barneby }\end{array}$ & $\begin{array}{l}\text { Arbusto, Árvore, } \\
\text { Subarbusto }\end{array}$ & B.V. Rabelo 2283 & NY, US \\
\hline Stylosanthes guianensis (Aubl.) Sw. & Erva, Subarbusto & S.A. Mori 15707 & HAMAB \\
\hline Stylosanthes humilis Kunth & Subarbusto & B.V. Rabelo 2273 & $\mathrm{MO}, \mathrm{NY}$ \\
\hline Swartzia racemosa Benth. & Árvore & B.V. Rabelo 2666 & NY \\
\hline
\end{tabular}


Research, Society and Development, v. 10, n. 13, e503101321290, 2021

(CC BY 4.0) | ISSN 2525-3409 | DOI: http://dx.doi.org/10.33448/rsd-v10i13.21290

\begin{tabular}{|c|c|c|c|c|}
\hline & Tephrosia sessiliflora (Poir.) Hassl. & Arbusto & S.A. Mori 15703 & HAMAB, K, NY \\
\hline & Zygia cataractae (Kunth) L.Rico & Árvore & $\begin{array}{l}\text { B.V. Rabelo 1427, } \\
1435\end{array}$ & HAMAB, K, NY \\
\hline & Zygia latifolia (L.) Fawc. \& Rendle & Arbusto, Árvore & B.V. Rabelo 1427 & NY \\
\hline Gentianaceae & Curtia tenuifolia (Aubl.) Knobl. & Erva & S.A. Mori 15750 & NY \\
\hline \multirow{2}{*}{ Heliconiaceae } & Heliconia psittacorum L.f. & Erva & S.A. Mori 15735 & K, NY, US \\
\hline & Heliconia spathocircinata Aristeg. & Erva & L.A. Pereira 83 & HAMAB \\
\hline Hydroleaceae & Hydrolea spinosa $\mathrm{L}$. & $\begin{array}{l}\text { Arbusto, Erva, } \\
\text { Subarbusto }\end{array}$ & B.V. Rabelo 496 & HAMAB, NY \\
\hline Lacistemataceae & Lacistema pubescens Mart. & Arbusto, Árvore & S.A. Mori 15757 & NY \\
\hline \multirow{5}{*}{ Lauraceae } & Cassytha filiformis $\mathrm{L}$. & Liana/volúvel/trepadeira & S.A. Mori 15740 & NY \\
\hline & Mezilaurus lindaviana Schwacke \& Mez & Arbusto, Árvore & B.V. Rabelo 2777 & NY \\
\hline & Ocotea bracteosa (Meisn.) Mez & Arbusto, Árvore & $\begin{array}{l}\text { B.V. Rabelo 2287, } \\
2490\end{array}$ & US, NY \\
\hline & Ocotea canaliculata (Rich.) Mez & Arbusto, Árvore & $\begin{array}{l}\text { B.V. Rabelo 2779, } \\
2258\end{array}$ & NY \\
\hline & Ocotea nigra Benoist & Arbusto, Árvore & B.V. Rabelo 2276 & NY \\
\hline \multirow{4}{*}{ Lecythidaceae } & $\begin{array}{l}\text { Eschweilera pedicellata (Rich.) } \\
\text { S.A.Mori }\end{array}$ & Árvore & $\begin{array}{l}\text { B.V. Rabelo } 2495 \\
2833,2844\end{array}$ & HAMAB, NY, US \\
\hline & Gustavia angustifolia Benth. & Árvore & B.V. Rabelo 1434 & HAMAB \\
\hline & Gustavia hexapetala (Aubl.) Sm. & Árvore & $\begin{array}{l}\text { B.V. Rabelo 2770, } \\
2783\end{array}$ & HAMAB \\
\hline & Lecythis pisonis Cambess. & Árvore & $\begin{array}{l}\text { B.V. Rabelo 2290, } \\
219\end{array}$ & HAMAB \\
\hline Lentibulariaceae & Utricularia subulata $\mathrm{L}$. & Erva & S.A. Mori 15717 & HAMAB, NY \\
\hline Loganiaceae & Spigelia hamelioides Kunth & Erva & B.V. Rabelo 1416 & HAMAB \\
\hline Malpighiaceae & Byrsonima crassifolia $($ L.) Kunth & Arbusto, Árvore & S.A. Mori 15741 & HAMAB, NY \\
\hline \multirow{4}{*}{ Malvaceae } & Apeiba albiflora Ducke & Árvore & B.V. Rabelo 2257 & HAMAB \\
\hline & Apeiba tibourbou Aubl. & Árvore & B.V. Rabelo 2257 & NY \\
\hline & Pavonia piptocalyx Krapov. & Arbusto, Subarbusto & B.V. Rabelo 1415 & HAMAB, NY \\
\hline & Waltheria indica $\mathrm{L}$. & Erva & S.A. Mori 15725 & HAMAB, NY \\
\hline Marantaceae & Ischnosiphon cannoideus L.Andersson & Erva & S.A. Mori 15738 & HAMAB \\
\hline \multirow{7}{*}{ Melastomataceae } & Bellucia grossularioides (L.) Triana & Árvore & B.V. Rabelo 2282 & NY \\
\hline & Clidemia sericea D. Don & Arbusto & S.A. Mori 15742 & $\begin{array}{c}\text { CAS, HAMAB, K, } \\
\text { NY }\end{array}$ \\
\hline & Miconia holosericea (L.) DC. & Arbusto & $\begin{array}{l}\text { R.L. Fróes s.n.; B.V. } \\
\text { Rabelo 2288; R.L. } \\
\text { Fróes } 27492 \\
\end{array}$ & US, $\mathrm{K}$ \\
\hline & Miconia regelii Cogn. & Arbusto & B.V. Rabelo 2487 & US \\
\hline & Miconia rufescens (Aubl.) DC. & Arbusto & S.A. Mori 15724 & HAMAB, K, NY \\
\hline & $\begin{array}{l}\text { Rhynchanthera serrulata (L.C.Rich.) } \\
\text { DC. }\end{array}$ & Erva & $\begin{array}{l}\text { S.A. Mori 15715, } \\
17395\end{array}$ & CAS, K, NY, US \\
\hline & Tibouchina aspera Aubl. & Subarbusto & S.A. Mori 15739 & NY \\
\hline Monimiaceae & Siparuna guianensis Aubl. & Arbusto, Árvore & R. Nonato 1 & HAMAB \\
\hline \multirow{2}{*}{ Moraceae } & $\begin{array}{l}\text { Brosimum guianense (Aubl.) Huber ex } \\
\text { Ducke }\end{array}$ & Árvore & B.V. Rabelo 2300 & NY \\
\hline & Ficus pakkensis Standl. & Árvore & B.V. Rabelo 2481 & NY \\
\hline Myristicaceae & Virola sebifera Aubl. & Árvore & B.V. Rabelo 2286 & INPA, NY \\
\hline Myrtaceae & $\begin{array}{l}\text { Campomanesia aromatica (Aubl.) } \\
\text { Griseb. }\end{array}$ & Árvore & $\begin{array}{l}\text { B.V. Rabelo 2845; } \\
\text { S.A. Mori } 17141 \\
17414\end{array}$ & $\begin{array}{l}\text { ASU, HAMAB, K, } \\
\text { NY, US }\end{array}$ \\
\hline
\end{tabular}




\begin{tabular}{|c|c|c|c|c|}
\hline & Eugenia biflora (L.) DC. & Arbusto, Árvore & $\begin{array}{l}\text { B.V. Rabelo 2278; } \\
\text { S.A. Mori } 15733\end{array}$ & $\begin{array}{l}\text { HAMAB, K, NY, } \\
\text { US }\end{array}$ \\
\hline & Eugenia flavescens DC. & Árvore & B.V. Rabelo 1045 & HAMAB \\
\hline & Eugenia omissa McVaugh & Árvore & B.V. Rabelo 2842 & HAMAB, K \\
\hline & Eugenia punicifolia (Kunth) DC. & Arbusto, Subarbusto & S.A. Mori 10570 & HAMAB, K, NY \\
\hline & Myrcia guianensis (Aubl.) DC. & Árvore & $\begin{array}{c}\text { S.A. Mori 15737, } \\
\text { 17416; B.V. Rabelo } \\
\text { 2514, 2229; J. } \\
\text { Mitchell 2229 }\end{array}$ & $\begin{array}{l}\text { HAMAB, K, NY, } \\
\text { US }\end{array}$ \\
\hline & Myrcia paivae O.Berg & Árvore & S.A. Mori 17420 & K, NY, SPF \\
\hline & $\begin{array}{l}\text { Myrciaria floribunda (H.West ex } \\
\text { Willd.) O.Berg }\end{array}$ & Árvore & $\begin{array}{l}\text { S.A. Mori 17412; } \\
\text { B.V. Rabelo 2274, } \\
2483\end{array}$ & $\begin{array}{l}\text { HAMAB, K, NY, } \\
\text { US }\end{array}$ \\
\hline & Psidium guineense $\mathrm{Sw}$. & Arbusto, Árvore & $\begin{array}{l}\text { S.A. Mori } 17404 \\
\text { B.V. Rabelo } 2512\end{array}$ & HAMAB, NY \\
\hline \multirow{21}{*}{ Orchidaceae } & Aspasia variegata Lindl. & Erva & P.C. Cantuária 2 & HAMAB \\
\hline & Brassavola martiana Lindl. & Erva & P.C. Cantuária 3 & HAMAB \\
\hline & Camaridium ochroleucum Lindl. & Erva & P.C. Cantuária 4 & HAMAB \\
\hline & Catasetum longifolium Lindl. & Erva & P.C. Cantuária 5 & HAMAB \\
\hline & $\begin{array}{l}\text { Catasetum macrocarpum Rich. ex } \\
\text { Kunth }\end{array}$ & Erva & P.C. Cantuária 1 & НАMАВ \\
\hline & $\begin{array}{l}\text { Dimerandra emarginata (G.Mey.) } \\
\text { Hoehne }\end{array}$ & Erva & P.C. Cantuária 6 & HAMAB \\
\hline & Epidendrum nocturnum Jacq. & Erva & P.C. Cantuária 8 & HAMAB \\
\hline & Epidendrum rigidum Jacq. & Erva & P.C. Cantuária 7 & HAMAB \\
\hline & Epidendrum strobiliferum Rchb.f. & Erva & P.C. Cantuária 9 & HAMAB \\
\hline & $\begin{array}{l}\text { Galeandra styllomisantha (Vell.) } \\
\text { Hoehne }\end{array}$ & Erva & P.C. Cantuária 11 & HAMAB \\
\hline & Habenaria trifida Kunth. & Erva & P.C. Cantuária 21 & HAMAB \\
\hline & Laelia gloriosa (Rchb.f.) L.O.Williams & Erva & P.C. Cantuária 10 & HAMAB \\
\hline & Lockhartia imbricata (Lam.) Hoehne & Erva & P.C. Cantuária 12 & HAMAB \\
\hline & $\begin{array}{l}\text { Polystachya concreta (Jacq.) Garay \& } \\
\text { H.R.Sweet }\end{array}$ & Erva & P.C. Cantuária 13 & НАMАВ \\
\hline & Polystachya foliosa (Hook.) Rchb.f. & Erva & P.C. Cantuária 14 & HAMAB \\
\hline & Scaphyglottis sickii Pabst & Erva & P.C. Cantuária 15 & HAMAB \\
\hline & Scaphyglottis stellata Lodd. ex Lindl. & Erva & P.C. Cantuária 16 & HAMAB \\
\hline & $\begin{array}{l}\text { Trichocentrum cebolleta (Jacq.) } \\
\text { M.W.Chase \& N.H.Williams } \\
\end{array}$ & Erva & P.C. Cantuária 17 & HAMAB \\
\hline & $\begin{array}{l}\text { Trigonidium acuminatum Bateman ex } \\
\text { Lindl. }\end{array}$ & Erva & P.C. Cantuária 18 & HAMAB \\
\hline & Vanilla cribbiana Soto Arenas & Erva & P.C. Cantuária 19 & HAMAB \\
\hline & $\begin{array}{l}\text { Vanilla palmarum (Salzm. ex Lindl.) } \\
\text { Lindl. }\end{array}$ & Erva & P.C. Cantuária 20 & HAMAB \\
\hline Passifloraceae & Passiflora foetida $\mathrm{L}$. & Liana/volúvel/trepadeira & B.V. Rabelo 488 & HAMAB \\
\hline \multirow{2}{*}{ Phyllanthaceae } & Amanoa guianensis Aubl. & Arbusto, Árvore & B.V. Rabelo 2784 & NY \\
\hline & Margaritaria nobilis L.f. & Arbusto & B.V. Rabelo 493 & NY \\
\hline Piperaceae & Piper marginatum Jacq. & Arbusto & B.V. Rabelo 1430 & HAMAB \\
\hline Plantaginaceae & Conobea aquatica Aubl. & Erva & S.A. Mori 17393 & HAMAB, K, NY \\
\hline \multirow{3}{*}{ Poaceae } & Andropogon bicornis $\mathrm{L}$. & Erva & S.A. Mori 15734 & NY \\
\hline & Andropogon leucostachyus Kunth & Erva & S.A. Mori 17397 & HAMAB, NY \\
\hline & Axonopus pubivaginatus Henrard & Erva & G.A. Black 9848 & IAN \\
\hline
\end{tabular}


Research, Society and Development, v. 10, n. 13, e503101321290, 2021

(CC BY 4.0) | ISSN 2525-3409 | DOI: http://dx.doi.org/10.33448/rsd-v10i13.21290

\begin{tabular}{|c|c|c|c|c|}
\hline & Axonopus purpusii $(\mathrm{Mez})$ Chase & Erva & $\begin{array}{l}\text { G.A. Black 9661, } \\
9664 \\
\end{array}$ & INPA, RB \\
\hline & Digitaria insularis (L.) Fedde & Erva & G.A. Black 9657 & IAN, INPA \\
\hline & Digitaria longiflora (Retz.) Pers. & Erva & G.A. Black 9674 & IAN \\
\hline & Eragrostis bahiensis Schrad. ex Nees & Erva & G.A. Black 9639 & IAN \\
\hline & Eragrostis ciliaris (L.) R.Br. & Erva & G.A. Black 9637 & IAN, INPA \\
\hline & Isachne polygonoides (Lam.) Döll & Erva & S.A. Mori 15736 & $\begin{array}{l}\text { HAMAB, K, NY, } \\
\text { UFRR }\end{array}$ \\
\hline & Leersia hexandra Sw. & Erva & E.M. Nowick 1002 & HAMAB \\
\hline & Leptochloa virgata (L.) P.Beauv. & Erva & G.A. Black 9636 & INPA \\
\hline & Panicum cayennense Lam. & Erva & G.A. Black 9659 & INPA \\
\hline & Panicum latissimum Spreng. & Erva & B.V. Rabelo 3317 & HAMAB \\
\hline & Panicum parvifolium Lam. & Erva & S.A. Mori 17398 & HAMAB \\
\hline & Panicum pilosum $\mathrm{Sw}$ & Erva & $\begin{array}{l}\text { S.A. Mori 17417; } \\
\text { B.V. Rabelo } 3319\end{array}$ & HAMAB \\
\hline & Panicum rudgei Roem. \& Schult. & Erva & $\begin{array}{l}\text { B.V. Rabelo 3321; } \\
\text { S.A. Mori } 15729\end{array}$ & HAMAB, NY \\
\hline & Paspalum conjugatum P.J.Bergius & Erva & G.A. Black 9647 & SP \\
\hline & $\begin{array}{l}\text { Paspalum melanospermum Desv. ex } \\
\text { Poir. } \\
\end{array}$ & Erva & G.A. Black 9671 & INPA \\
\hline & Rugoloa pilosa (Sw.) Zuloaga & Erva & S.A. Mori 17417 & MO \\
\hline & Setaria tenax (Rich.) Desv. & Erva & $\begin{array}{l}\text { B.V. Rabelo 2268; } \\
\text { S.A. Mori } 15730\end{array}$ & HAMAB, NY \\
\hline & Streptostachys asperifolia Desv. & Erva & $\begin{array}{l}\text { S.A. Mori 17402; } \\
\text { B.V. Rabelo } 3320\end{array}$ & НАMAB \\
\hline & Trachypogon spicatus (L.f.) Kuntze & Erva & $\begin{array}{l}\text { S.A. Mori 17396, } \\
15746 \\
\end{array}$ & HAMAB, K, NY \\
\hline Polygalaceae & Polygala trichosperma $\mathrm{L}$. & Erva & S.A. Mori 15744 & NY \\
\hline & Ardisia guianensis (Aubl.) Mez & Arbusto & B.V. Rabelo 1414 & HAMAB \\
\hline Primulaceae & Cybianthus prieurii A.DC. & Arbusto, Árvore & B.V. Rabelo 2292 & $\begin{array}{l}\text { HAMAB, MO, } \\
\text { NY }\end{array}$ \\
\hline Rubiaceae & Alibertia edulis (Rich.) A.Rich. & Arbusto, Árvore & $\begin{array}{l}\text { S.A. Mori 15756; } \\
\text { J.R. Lima-Barbosa } \\
142 \\
\end{array}$ & $\mathrm{NY}, \mathrm{RB}$ \\
\hline Salicaceae & Casearia sylvestris $\mathrm{Sw}$. & $\begin{array}{l}\text { Arbusto, Árvore, } \\
\text { Subarbusto }\end{array}$ & S.A. Mori 15709 & HAMAB, NY \\
\hline Conindocos & Cupania oblongifolia Mart. & Árvore & B.V. Rabelo 2488 & HAMAB, NY \\
\hline sapinuacede & Paullinia pinnata $\mathrm{L}$. & Liana/volúvel/trepadeira & B.V. Rabelo 1423 & HAMAB \\
\hline Sematophyllaceae & $\begin{array}{l}\text { Sematophyllum subsimplex (Hedw.) } \\
\text { Mitt. }\end{array}$ & Erva & $\begin{array}{l}\text { S.A. Mori 17407, } \\
17408 \\
\end{array}$ & HAMAB, NY \\
\hline Simaroubaceae & Simarouba amara Aubl. & Árvore & D.F. Austin 7028 & HAMAB \\
\hline & Capsicum anпиum $\mathrm{L}$. & Erva & $\begin{array}{c}\text { L.A. Pereira 1822, } \\
\text { 1824, 1899, 1900, } \\
\text { 1903; L.E. } \\
\text { Nascimento 07, } 09\end{array}$ & $\mathrm{RB}$ \\
\hline & Capsicum baccatum $\mathrm{L}$. & Erva & L.A. Pereira 1819 & $\mathrm{RB}$ \\
\hline Solanaceae & Capsicum chinense Jacq. & Erva & $\begin{array}{l}\text { L.A. Pereira 1815, } \\
\text { 1816, 1817, 1819, } \\
\text { 1820, 1821, 1822, } \\
\text { 1823, 1825, 1873, } \\
\text { 1901, 1902, 1904, } \\
\text { 1905, 1906, 1908; } \\
\text { L.E. Nascimento } 13\end{array}$ & $\mathrm{BHCB}, \mathrm{CEN}, \mathrm{RB}$ \\
\hline & Capsicum frutescens $\mathrm{L}$. & Erva & L.A. Pereira 1820 & $\mathrm{RB}$ \\
\hline
\end{tabular}




\begin{tabular}{|l|l|c|c|c|}
\hline Turneraceae & Turnera brasiliensis Willd. ex Schult. & Erva, Subarbusto & S.A. Mori 15731 & K, NY \\
\hline Vochysiaceae & Salvertia convallariodora A. St.-Hil. & Árvore & S.A. Mori 15720 & HAMAB, NY, US \\
\hline \multirow{3}{*}{ Xyridaceae } & Xyris jupicai Rich. & Erva & $\begin{array}{r}\text { S.A. Mori 15713, } \\
17399 ; \text { R. Nonato } \\
25\end{array}$ & HAMAB, NY \\
\cline { 2 - 5 } & Xyris laxifolia Mart. & Erva & $\begin{array}{c}\text { L.A. Pereira 63; } \\
\text { S.A. Mori 17399 }\end{array}$ & HAMAB, NY \\
\hline
\end{tabular}

Fonte: Autores (2021).

O destaque para a família Fabaceae (ou Leguminosae) pode ter relação com a constatação de que esta é a com maior diversidade e número de espécies na flora brasileira, estando entre as três famílias mais diversas em todos os domínios fitogeográficos do Brasil (Lima et al., 2015). Leguminosae compreende 795 gêneros e cerca de 20.000 espécies, se configurando entre as três maiores famílias, conforme Lewis et al. (2005), Lima et al. (2015) e Legume Phylogeny Working Group (LPWG, 2017). Estima-se que cerca de 50\% das espécies são endêmicas do país e dentre os gêneros representados no Brasil, 25 apresentam apenas espécies cultivadas alimentícias (Cicer L. e Glycine Willd.), ornamentais (Delonix Raf., Adenanthera Speg. e Tipuana (Benth.) Benth.) ou com outros usos (Lima et al., 2015).

De acordo com Lima et al. (2015), as espécies desta família de plantas destacam-se pela importância ecológica, em decorrência da associação da maioria de seus representantes com bactérias fixadoras de nitrogênio, tornando-se as principais responsáveis pela entrada do nitrogênio nos ecossistemas terrestres. Atualmente a família é dividida em seis subfamílias (Cercidoideae, Caesalpinioideae, Dialioideae, Detarioideae, Duparquetioideae e Papilionoideae), das quais apenas Duparquetioideae, composta somente pela espécie Duparquertia orchidacea Baill., não ocorre no Brasil (LPWG, 2017).

Diversos autores têm destacado a importância das Fabaceae na composição e riqueza florística. Oliveira et al. (2008) realizaram estudo da composição florística e estrutural na Amazônia Central, onde foram registrados 670 indivíduos pertencentes a 48 famílias e 245 espécies, em que as Fabaceae se destacaram em relação à riqueza de espécies. Carim et al. (2008), estudando a composição florística e a estrutura de uma floresta de várzea no Município de Mazagão, Amapá, verificaram que Fabaceae foi a família com o maior número de espécies, evidenciando alta similaridade florística e baixa diversidade de espécies.

Pereira et al. (2011) realizaram estudo sobre a florística e a estrutura de uma porção da vegetação arbórea da Reserva de Desenvolvimento Sustentável do Rio Iratapuru no Estado do Amapá, onde registraram 101 espécies arbóreas dividas em 32 famílias, sendo Fabaceae a que apresentou maior riqueza específica (24) e abundância (105). Carim et al. (2013) discorreram acerca da fitossociologia e florística de um fragmento de Floresta Ombrófila Densa no Amapá, com destaque para as famílias Fabaceae e Lauraceae em relação à riqueza e frequência. Em estudo realizado no arquipélago de Marajó/PA, após inventariar a área e analisar a distribuição florística das Leguminosae, Silva et al. (2013), observaram a ocorrência de 121 espécies.

O estudo de Silva (2014) verificou a composição florística em um trecho de floresta de terra firme no sudoeste do Estado do Amapá e destacou predominância da família Fabaceae (20,33\%). Em sua pesquisa, Moraes (2016) discorreu sobre a fitossociologia de uma floresta de várzea no Estado do Amapá, onde 2.050 indivíduos foram amostrados e a família mais representativa em número de espécies foi Fabaceae (16).

Cantuária et al. (2017), ao analisar a florística da família em um trecho de floresta de várzea na APA da Fazendinha, observaram que os gêneros com maior frequência foram Mora Benth., com 31,91\%, Pentaclethra Benth., com 27,66\%, e Pterocarpus Jacq., com 19,15\%. Cantuária et al. (2021) registraram a ocorrência da família na APA da Fazendinha, ressaltando a importância das Fabaceae para a região, uma vez que colabora expressamente para biodiversidade das floretas tropicais, bem como para o equilíbrio de seus ecossistemas. 
Obter conhecimento acerca dessa família é essencial tanto para a florística quanto socioeconomicamente, uma vez que muitas espécies desta família servem como fonte de renda para as comunidades, como ornamentação ou na produção de medicamentos, contribuindo para a manutenção da diversidade (Cantuária et al., 2021; Lima et al., 2015).

No que tange aos pesquisadores que fizeram coletas na área de abrangência deste estudo, registraram-se 19 coletores. Destacam-se B.V. Rabelo, com o maior número de coletas (187), seguido de S.A. Mori (148), L.A. Pereira (50), P.C. Cantuária (21), G.A. Black (13), R. Nonato (11), D.C. Daly (10) e R.L. Fróes (9), conforme a Tabela 1.

$\mathrm{Na}$ a análise da evolução temporal das coletas realizadas, é interessante notar que estas ocorreram em um período que compreende desde a década de 1950 à década de 2016, mostrando a existência de informações consistentes acerca de coletas efetuadas nessa área. A década de 1980 correspondeu ao período com o maior número de coletas $(378=75,44 \%)$ e o ano de 1983 com o valor mais expressivo de registros $(204=53,96 \%)$, de acordo com a Figura 2.

Figura 2 - Número de coletas de plantas por ano na Bacia Hidrográfica do Igarapé da Fortaleza, AP-010, Amapá, 2021.

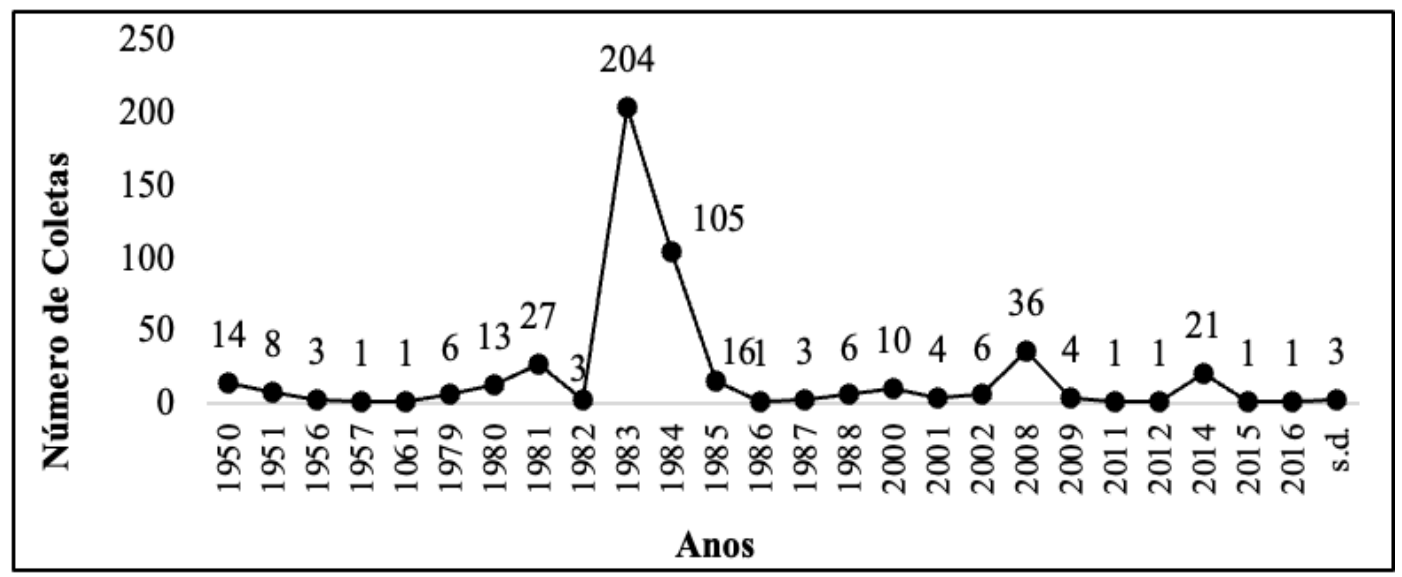

Fonte: Autores (2021).

Nota-se, a partir da análise temporal das coletas, que esse destaque tem relação com o fato de, basicamente, as informações sobre a flora do Amapá serem fruto das expedições científicas realizadas de 1961 a 1963 (Pires, 1963 ), por meio de cooperação entre o Instituto de Pesquisa Agropecuária do Norte, Museu Paraense Emílio Goeldi, New York Botanical Garden e Museu de História Natural Ângelo Moreira Costa Lima. Além das expedições lideradas por D.F. Austin, em 1979 (Austin, 1981), S.A. Mori e D. Daly, em 1981 (Mori et al., 1989) e G.T. Prance, em 1983 (Prance, 1997).

Do total de espécies coletadas na Bacia Hidrográfica do Igarapé da Fortaleza, 106 (32,12\%) estão classificadas quanto à forma de vida como ervas (Figura 3). Essa constatação reforça as características da área, que é formada por distintos ambientes, mas principalmente por áreas de ressaca, onde predominantemente a vegetação herbácea é dominante. 
Figura 3 - Classificação das formas de vida relacionadas ao número de espécies registradas na Bacia Hidrográfica do Igarapé da Fortaleza, AP-010, Amapá, 2021.

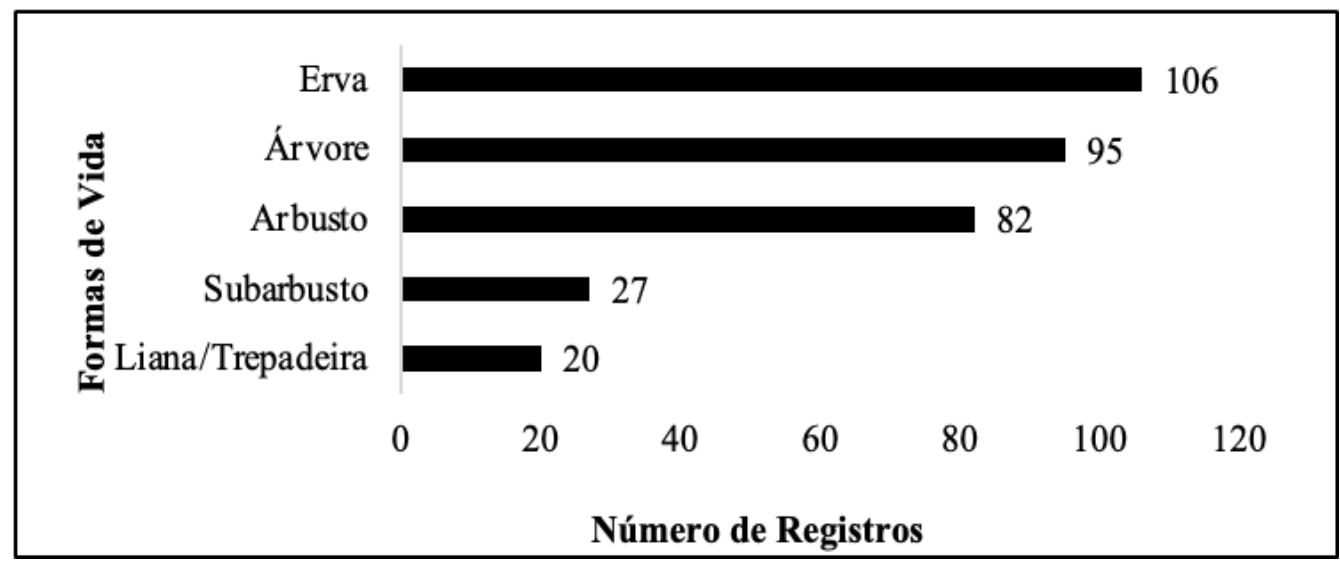

Fonte: Autores (2021).

As espécies dominantes nas áreas de ressaca são: Eleocharis intesticta (Vahl) Roem. e Schult, Thalia geniculata L., Sagittaria rhombifolia Cham., Montrichardia arborescens Schott. e Mauritia flexuosa L. f. As formas biológicas mais frequentes $(78 \%)$ foram as anfíbias e/ou emergentes. Os autores destacam também outras informações, tais como as épocas de floração e frutificação de várias espécies. De acordo com a distribuição destas, foram verificadas diferenças e similaridades entre as áreas de ressaca estudadas, nas quais notaram-se variações entre a vegetação das ressacas preservadas e as impactadas pela atividade humana (Costa Neto et al., 2004).

As áreas de ressaca correspondem a ambientes caracterizados por bacias de acumulação de água influenciadas pelas chuvas, marés e rios, por meio de canais (Takiyama et al., 2004). Costa Neto et al. (2004) identificaram na Bacia Hidrográfica do Igarapé da Fortaleza um total de 119 espécies, distribuídas em 44 famílias e 83 gêneros. A família com maior diversidade foi Cyperaceae, com 18 espécies, seguida da família Poaceae, com 15, Fabaceae, com sete, e Rubiaceae, com três.

Diante dessa perspectiva, os trabalhos sobre a florística de um local são tão importantes quanto as informações por eles geradas. É consensual que os herbários também são indispensáveis para estudos de plantas e constituem suporte à pesquisa para diversas áreas do conhecimento. Além de documentar a diversidade biológica do país, os espécimes depositados em herbários guardam parte da história de regiões anteriormente cobertas por vegetação natural e hoje ocupadas por cidades, empreendimentos diversos ou áreas hoje desflorestadas (Peixoto \& Barbosa, 2002; Peixoto et al., 2009).

Considera-se que as coleções botânicas são reconhecidas, não apenas pelos cientistas, como prioritárias para se planejar estudos de biodiversidade, manejo sustentável dos recursos naturais, programas de recuperação ambiental, ecoturismo e outros (Peixoto et al., 2009; Peixoto \& Morim, 2003). Portanto, as coleções biológicas continuam sendo a principal fonte para estudos sobre diversidade biológica (Bicudo \& Shepherd, 1998).

Dessa forma trabalhos que destacam as informações contidas em coleções biológicas são importantes, pois aumentam a visibilidade e a relevância das coleções e de pesquisas nelas realizadas, como consequência, a exposição pública dos dados permite melhora na sua qualidade. Entretanto, talvez o benefício preponderante do compartilhamento de dados seja a aceleração no avanço científico com o acesso aos dados e a possibilidade de maior integração entre diferentes áreas de conhecimento (Peixoto et al., 2009). 


\section{Considerações Finais}

Conclui-se que a diversidade florística da Bacia Hidrográfica do Igarapé da Fortaleza é bastante significativa, com destaque para a família Fabaceae, com o maior número de registros, seguida de Poaceae e Orchidaceae, sendo as plantas herbáceas as mais representativas. A maior parte das coletas foi efetuada na década de 1980, período importante de grandes projetos de levantamento florístico que foram desenvolvidos na Amazônia. O coletor mais destacado foi Benedito Vitor Rabelo, que fundou o Herbário Amapaense (HAMAB) em 1979. O HAMAB corresponde à coleção que detém o maior número de amostras, mas há material depositado somente em herbários internacionais.

Considera-se que mesmo com expressiva diversidade registrada, na Bacia Hidrográfica do Igarapé da Fortaleza ainda há necessidade de estudos adicionais, para a coleta de espécies ainda não registradas que podem substanciar a tomada de decisões ambientais.

Preencher essas lacunas existentes no conhecimento da cobertura vegetal da região e a organização e interpretação de dados de coletas botânicas herborizadas no HAMAB e em outros herbários são fundamentais, uma vez que conhecer a composição florística, sua diversidade e distribuição permite desenvolver ações de conservação de uma região, desenvolver potenciais de uso sustentável, bem como incentivar novas descobertas.

Portanto estudos baseados em bancos de dados se constituem como elementos importantes na compreensão da composição florística de ecossistemas e como subsídio para a determinação de ações políticas e ambientais para a Bacia Hidrográfica do Igarapé da Fortaleza.

\section{Referências}

Abreu, J. C., Guedes, M. C., Lira Guedes, A. C., \& Batista, E. M. (2014). Estrutura e distribuição espacial de andirobeiras (Carapa spp.) em floresta de várzea do estuário amazônico. Ciência Florestal, 24(4), 1007-1016. https://periodicos.ufsm.br/cienciaflorestal/article/view/16614/pdf.

Angiosperm Phylogeny Group. (2016). An update of the Angiosperm Phylogeny Group classification for the orders and families of flowering plants: APG IV. Botanical Journal of the Linnean Society, 181(1), 1-20. https://academic.oup.com/botlinnean/article/181/1/1/2416499.

Austin, D. F. (1981). Novidades nas Convolvulaceae da flora amazônica. Acta Amazonica, 11(1), 291-295. https://www.scielo.br/j/aa/a/mqjFd3JB 7htjxFKSTMbv7Hy/?lang=pt.

Bicudo, C. E. M., \& Shepherd, G. J. Síntese. In: Bicudo, C. E. M., \& Shepherd, G. J. (1998). Fungos, microrganismos e plantas: Biodiversidade do Estado de São Paulo, Brasil (pp. 77-79). Fapesp.

Cantuária, Patrick C., Alves, C. M. G., Medeiros, T. D. S., Silva, R. B. L., Freitas, J. L., Cantuária, M. F., \& Cantuária, Pablo C. (2017). Ocorrência de Fabaceae da Área de Proteção Ambiental da Fazendinha, Macapá, Amapá, Brasil. Biota Amazônia, 7(2), 49-52. https://periodicos.unifap.br/index.php/biota/article/view/2993/v7n2p49-52.pdf.

Cantuária, Patrick. C., Medeiros, T. D. S., Silva, R. B. L., Cantuária, M. F., Cantuária, Pablo. C., Costa Neto, S. V., \& Negrão, M. F. (2021). Flora da Área de Proteção Ambiental da Fazendinha (Amapá): Orchidaceae. Biota Amazônia, 11(1), 52-59. https://periodicos.unifap.br/index.php/biota/article/view/3777.

Carim, M. J. V., Jardim, M. A. G., \& Medeiros, T. D. S. (2008). Composição florística e estrutura de floresta de várzea no município de Mazagão, Estado do Amapá, Brasil. Scientia Forestalis, 36(79), 191-201. https://www.ipef.br/publicacoes/scientia/nr79/cap03.pdf.

Carim, M. J. V., Guillaumet, J. -L. B., Guimarães, J. R. S., \& Tostes, L. C. L. (2013). Composição e estrutura de floresta ombrófila densa do extremo norte do Estado do Amapá, Brasil. Biota Amazônia, 3(2), 1-10. https://periodicos.unifap.br/index.php/biota/article/view/610/v3n2p1-10.pdf.

Conservação Internacional Brasil. (2009). Corredor da Biodiversidade do Amapá. Governo do Estado do Amapá, Secretaria do Estado do Meio Ambiente do Amapá. https://www.conservation.org/docs/default-source/brasil/corredor_do_amapa.pdf.

Costa Neto, S. V., Tostes, L. C. L., \& Thomaz, D. O. Inventário florístico das ressacas das Bacias do Igarapé da Fortaleza e do Rio Curiaú. In: Takiyama, L. R., \& Silva, A. Q. (2004). Diagnóstico das ressacas do Estado do Amapá: Bacias do Igarapé da Fortaleza e Rio Curiaú, Macapá-AP (pp. 1-22). CPAq/IEPA e DGEO/SEMA. http://www.iepa.ap.gov.br/arquivopdf/livro_ressacas/CAPITULO_01.pdf.

Cunha, A. C., Cunha, H. F. A., Brasil Júnior, A. C. P., Daniel, L. A., \& Schulz, H. E. (2004). Qualidade microbiológica da água em rios de áreas urbanas e periurbanas no baixo Amazonas: o caso do Amapá. Engenharia Sanitária e Ambiental, 9(4), 322-328. https://www.scielo.br/j/esa/a/XffprqR4BtymvL4QnSYvxZg/?lang=pt.

Flora do Brasil 2020. (2021). Flora do Brasil 2020. Jardim Botânico do Rio de Janeiro. http://floradobrasil.jbrj.gov.br/.

Instituto Brasileiro de Geografia e Estatística. (2012). Manual técnico da vegetação brasileira. IBGE. https://biblioteca.ibge.gov.br/visualizacao/ monografias/GEBIS\%20-\%20RJ/ManuaisdeGeociencias/Manual\%20Tecnico\%20da\%20Vegetacao\%20Brasileira\%20n.1.pdf. 
Legume Phylogeny Working Group. (2017). A new subfamily classification of the Leguminosae based on a taxonomically comprehensive phylogeny. Taxon, 66(1), 44-77. https://onlinelibrary.wiley.com/doi/10.12705/661.3.

Lewis, G. P., Schrire, B., Mackinder, B., \& Lock, M. (2005). Legumes of the World. Royal Bot. Gard. Kew.

Lima, H. C., Queiroz, L. P., Morim, M. P., Souza, V. C., Dutra, V. F., Bortoluzzi, R. L. C., \& Cordula, E. (2015). Fabaceae in Lista de Espécies da Flora do Brasil. Jardim Botânico do Rio de Janeiro. http://floradobrasil.jbrj.gov.br/jabot/floradobrasil/FB115.

Moraes, L. L. C. M. (2016). Fitossociologia de uma floresta de várzea e de igapó na planície de inundação do Rio Maracá, Amapá, Amazônia Oriental. (Dissertação de Mestrado, Universidade Federal do Amapá, Macapá, Brasil). http://repositorio.unifap.br/jspui/handle/123456789/488.

Mori, S. A., Rabelo, B. V., Tsou, C. H., \& Daly, D. (1989). Composition and structure of an eastern Amazonian forest at Camaipi, Amapá, Brazil. Boletim do Museu Paraense Emílio Goeldi, Série Botânica, 5(1), 3-18. https://agris.fao.org/agris-search/search.do?recordID=BR9208037.

Neves, D. G. (2012). Influência da vegetação na precipitação pluviométrica sazonal do Estado do Amapá: Um estudo de sensibilidade climática. (Dissertação de Mestrado, Universidade Federal do Amapá, Macapá, Brasil). http://repositorio.unifap.br/jspui/handle/123456789/488.

Oliveira, A. N., Amaral, I. L., Ramos, M. B. P., Nobre, A. D., Couto, L. B., \& Sahdo, R. M. (2008). Composição e diversidade florístico estrutural de um hectare de floresta densa de terra firme na Amazônia Central, Amazonas, Brasil. Acta Amazonica, 38(4), 627-641. https://doi.org/10.1590/S004459672008000400005

Peixoto, A. L., Barbosa, M. R. V., Canhos, D. A. L., \& Maia, L. C. Coleções botânicas: objetos e dados para a ciência. In: Granato, M., \& Rangel, M. (2009). Cultura Material e Patrimônio da Ciência e Tecnologia. Museu da Astronomia e Ciências Afins. http://inct.florabrasil.net/wpcontent/uploads/downloads/2010/04/colec\%CC\%A7o\%CC\%83es-botanicas-capitulo-2009.pdf.

Peixoto, A. L., \& Barbosa., M. R. V. (2002). "The currente situation in Brazil: general strategies, regional differences, local floras, state-level floras and herbarium databasing". http://www.cria.org/htm.

Peixoto, A. L., \& Morim, M. P. (2003). Coleções botânicas: Documentação da biodiversidade brasileira. Ciência \& Cultura, 3(55), 21-48. http://cienciaecultura.bvs.br/pdf/cic/v55n3/a16v55n3.pdf.

Pereira, L. A., Sobrinho, F. A. P., \& Costa Neto, S. V. (2011). Florística e estrutura de uma mata de terra firme na Reserva de Desenvolvimento Sustentável Rio Iratapuru, Amapá, Amazônia Oriental, Brasil. Floresta, 41(1), 113-122. https://revistas.ufpr.br/floresta/article/download/21191/13979.

Pires, J. M. (1963). Plantas do Amapá. Instituto de Pesquisa Agropecuária do Norte.

Prance, G. T. (1997). Floristic inventory of the tropics: where do we stand? Annals of the Missouri Botanical Garden, 64, 559-684 https://www.biodiversitylibrary.org/part/40267.

Silva, W. A. S., Carim, M. J. V., Guimarães, J. R. S., \& Tostes, L. C. L. (2014). Composição e Diversidade florística em um trecho de floresta de Terra firme no Sudoeste do Estado do Amapá, Amazônia Oriental, Brasil. Biota Amazônia, 4(3), 31-36. https://periodicos.unifap.br/index.php/biota/article/view/859.

Silva, W. L. S. E., Gurgel, E. S. C., Santos, J. U. M., \& Silva, M. F. (2013). Inventário e distribuição geográfica de Leguminosae no arquipélago de Marajó, PA, Brasil. Hoehnea, 40(4), 627-647. https://www.scielo.br/j/hoehnea/a/Yc9mhtPgbKVCP7nZc6VjYsN/?format=pdf\&lang=pt.

Siqueira, A. A., Santos Filho, J. V., \& Siqueira Filho, J. A. (2012). Carolus: um novo sistema gratuito para manipulação eletrônica de coleções botânicas. Rodriguésia, 63(3), 715-732, 2012. https://www.scielo.br/j/rod/a/rgcWzmwNDF6vRYFTRg3xT5Q/?lang=pt.

SpeciesLink network (2021). https://specieslink.net/search.

Takiyama, L. R., Silva, A. Q., Costa, W. S. P., \& Nascimento, H. S. Qualidade das águas das ressacas das bacias do Igarapé da Fortaleza e do Rio Curiaú. In: Takiyama, L. R., \& Silva, A. Q. (2004). Diagnóstico de ressacas do Estado do Amapá: Bacias do Igarapé da Fortaleza e do Rio Curiaú (pp. 99-122) GEA/SETEC/IEPA. http://www.iepa.ap.gov.br/metadados/instituicoes/iepa/projetos/ressacas/documentos/6finalQUALIDADE\%20DE\%20AGUA.pdf.

Takiyama, L. R., Silva, U. R. L., Jimenez, E. A., Pereira, R. A., Zacardi, D. M., Fernandes, E. F., \& Santos, V. F. (2012). Projeto zoneamento ecológico econômico urbano das áreas de ressacas de Macapá e Santana, estado do Amapá: relatório técnico final. IEPA. https://www.mpap.mp.br/images/PRODEMAC/livros/Livro\%20Ressacas.pdf.

Thiers, B. (2021). Index Herbariorum: A global directory of public herbaria and associated staff. New York Botanical Garden's Virtual Herbarium. http://sweetgum.nybg.org/science/ih/.

Tropicos.org. (2021). Missouri Botanical Garden. https://tropicos.org. 